\title{
Node Centrality for Continuous-Time Quantum Walks
}

\author{
Luca Rossi ${ }^{1}$, Andrea Torsello ${ }^{2}$, and Edwin R. Hancock ${ }^{3}$ \\ 1 School of Computer Science, University of Birmingham, UK \\ 2 Department of Environmental Science, Informatics, and Statistics, \\ Ca' Foscari University of Venice, Italy \\ 3 Department of Computer science, University of York, UK
}

\begin{abstract}
The study of complex networks has recently attracted increasing interest because of the large variety of systems that can be modeled using graphs. A fundamental operation in the analysis of complex networks is that of measuring the centrality of a vertex. In this paper, we propose to measure vertex centrality using a continuous-time quantum walk. More specifically, we relate the importance of a vertex to the influence that its initial phase has on the interference patterns that emerge during the quantum walk evolution. To this end, we make use of the quantum Jensen-Shannon divergence between two suitably defined quantum states. We investigate how the importance varies as we change the initial state of the walk and the Hamiltonian of the system. We find that, for a suitable combination of the two, the importance of a vertex is almost linearly correlated with its degree. Finally, we evaluate the proposed measure on two commonly used networks.
\end{abstract}

Keywords: Vertex Centrality, Complex Network, Quantum Walk, Quantum Jensen-Shannon Divergence.

\section{Introduction}

In recent years, an increasing number of researchers have turned their attention to the study complex networks 1]. Complex network are ubiquitous in a large number of real-world systems. A non-exhaustive list of examples includes metabolic networks [2], protein interactions [3], brain networks [4] and scientific collaboration networks [5]. A fundamental task in complex network analysis is that of measuring the centrality of a vertex, i.e., its importance. To this end, a number of centrality indices have been introduced in the literature [1, [6 6 ]. Each of these captures different but equally significant aspects of vertex importance.

Perhaps the most intuitive centrality measure is degree centrality [7]. This is defined as the number of links incident upon a node, i.e., the degree of the node. The degree centrality naturally interprets the number of edges incident on a vertex as a measure of its "popularity", or, alternatively, as the risk of a node being infected in an epidemiological scenario. Closeness centrality [10], on the other hand, links the importance of a vertex to its proximity to the remaining vertices 
of the graph. More precisely, the closeness centrality is defined as the inverse of the sum of the distance of a vertex to the remaining nodes of the graph, i.e., $C C(u)=\frac{n-1}{\sum_{v=1}^{n} d(u, v)}$ where $d(u, v)$ denotes the shortest path distance between nodes $u$ and $v$. The betweenness centrality [7] is a measure of the extent to which a given vertex lies on the paths between the remaining vertices, where the path may be either that of shortest length or a random walk between the nodes. If $s p\left(v_{1}, v_{2}\right)$ denotes the number of shortest paths from node $v_{1}$ to node $v_{2}$, and $s p\left(v_{1}, u, v_{2}\right)$ denotes the number of shortest paths from $v_{1}$ to $v_{2}$ that pass through node $u$, the betweenness centrality of $u$ is $B C(u)=\sum_{v_{1}=1}^{n} \sum_{v_{2}=1}^{n} \frac{s p\left(v_{1}, u, v_{2}\right)}{s p\left(v_{1}, v_{2}\right)}$. Note that this definition assumes that the communication takes place along the shortest path between two vertices. A number of measures have been introduced to account for alternative scenarios in which the information is allowed to flow through different paths [1, 6, 8].

Recently, there has also been a surge of interest in using quantum walks as a primitive for designing novel quantum algorithms on graph structures [11]. Quantum walks on graphs represent the quantum mechanical analogue of the classical random walk on a graph. Despite being similar in their definition, the dynamics of the two walks can be remarkably different. In the classical case the evolution of the walk is governed by a double stochastic matrix, while in the quantum case the evolution is governed by a unitary matrix, thus rendering the walk reversible and non-ergodic. Moreover, the state vector of the classical random walk is real-valued, while in the quantum case the state vector is complex-valued. As there is no constraint on the sign and phase of the amplitudes, different paths are allowed to interfere with each other in both constructive and destructive ways. This in turn gives rise to faster hitting times and reduces the problems of tottering observed in classical random walks [11].

In this paper, we propose to measure the centrality of a vertex using a continuous-time quantum walk. More specifically, we relate the importance of a vertex to the influence that its initial phase has on the evolution of a suitably defined quantum walk. To this end, we make use of the quantum Jensen-Shannon divergence, a recently introduced generalisation of the classical Jensen-Shannon divergence to quantum states [12]. Just as the classical Jensen-Shannon divergence [13], the quantum Jensen-Shannon divergence is symmetric, bounded and always defined. From a physical perspective, the QJSD is computed from density matrices, whose entries are observables. As a consequence, it should be possible, at least in theory, to design a quantum algorithm to compute the QJSD centrality that could benefit from the power of quantum computers. However, the design of such an algorithm is beyond the scope of this paper.

The remainder of this paper is organised as follows: Section 2 provides an essential introduction to the basic terminology required for understanding the proposed quantum mechanical framework. With these notions to hand, we introduce our centrality measure in Section 3 and we study its properties. In Section 4 we apply the proposed measure to the analysis of two commonly used network models, while the conclusions are presented in Section 5. 


\section{Quantum Mechanical Background}

The continuous-time quantum walk [14] is a natural quantum analogue of the classical random walk. Given a graph $G=(V, E)$, classical random walks model a diffusion process over the node set $V$, and have proven to be a useful tool in the analysis of its structure. Similarly, the continuous-time quantum walk is defined as a dynamical process over the vertices of the graph. By contrast to the classical case, where the state vector is constrained to lie in a probability space, in the quantum case the state of the system is defined through a vector of complex amplitudes over the node set $V$ whose squared norm sums to unity over the nodes of the graph, with no restriction on their sign or complex phase. These phase differences allow interference effects to take place. Moreover, in the quantum case the evolution of the state vector of the walker is governed by a complex valued unitary matrix, whereas the dynamics of the classical random walk is governed by a stochastic matrix. Hence the evolution of the quantum walk is reversible, implying that quantum walks are non-ergodic and do not possess a limiting distribution. As a result, the behaviour of classical and quantum walks differs significantly, and quantum walks possess a number of interesting properties not exhibited by classical random walks.

More formally, using the Dirac notation, we denote the basis state corresponding to the walk being at vertex $u \in V$ as $|u\rangle$. A general state of the walk is a complex linear combination of the basis states, such that the state of the walk at time $t$ is defined as

$$
\left|\psi_{t}\right\rangle=\sum_{u \in V} \alpha_{u}(t)|u\rangle
$$

where the amplitude $\alpha_{u}(t) \in \mathbb{C}$ and $\left|\psi_{t}\right\rangle \in \mathbb{C}^{|V|}$ are both complex.

At each instant in time the probability of the walker being at a particular vertex of the graph is given by the square of the norm of the amplitude of the relative state. Let $X^{t}$ be a random variable giving the location of the walker at time $t$. Then the probability of the walker being at the vertex $u$ at time $t$ is given by $\operatorname{Pr}\left(X^{t}=u\right)=\alpha_{u}(t) \alpha_{u}^{*}(t)$, where $\alpha_{u}^{*}(t)$ is the complex conjugate of $\alpha_{u}(t)$. Moreover $\sum_{u \in V} \alpha_{u}(t) \alpha_{u}^{*}(t)=1$ and $\alpha_{u}(t) \alpha_{u}^{*}(t) \in[0,1]$, for all $u \in V$, $t \in \mathbb{R}^{+}$.

The evolution of the walk is then given by the Schrödinger equation, where we take the time-independent Hamiltonian of the system to be the graph Laplacian, yielding

$$
\frac{\partial}{\partial t}\left|\psi_{t}\right\rangle=-i L\left|\psi_{t}\right\rangle
$$

Given an initial state $\left|\psi_{0}\right\rangle$, we can solve Eq. (2) to determine the state vector at time $t$

$$
\left|\psi_{t}\right\rangle=e^{-i L t}\left|\psi_{0}\right\rangle
$$

Note that generally one may use any Hermitian operator as the Hamiltonian. Common choices are the graph adjacency matrix, the normalised Laplacian and the signless Laplacian. 
Finally, we can compute the spectral decomposition of the graph Laplacian $L=\Phi \Lambda \Phi^{\top}$, where $\Phi$ is the $n \times n$ matrix $\Phi=\left(\phi_{1}\left|\phi_{2}\right| \ldots\left|\phi_{j}\right| \ldots \mid \phi_{n}\right)$ with the ordered eigenvectors $\phi_{j}$ s of $L$ as columns and $\Lambda=\operatorname{diag}\left(\lambda_{1}, \lambda_{2}, \ldots, \lambda_{j}, \ldots, \lambda_{n}\right)$ is the $n \times n$ diagonal matrix with the ordered eigenvalues $\lambda_{j}$ of $L$ as elements, such that $0=\lambda_{1} \leq \lambda_{2} \leq \ldots \leq \lambda_{n}$. Using the spectral decomposition of the graph Laplacian and the fact that $\exp [-i L t]=\Phi \exp [-i \Lambda t] \Phi^{\top}$ we can then write

$$
\left|\psi_{t}\right\rangle=\Phi e^{-i \Lambda t} \Phi^{\top}\left|\psi_{0}\right\rangle
$$

\subsection{Quantum Jensen-Shannon Divergence}

The density operator (or density matrix) is introduced in quantum mechanics to describe a system whose state is an ensemble of pure quantum states $\left|\psi_{i}\right\rangle$, each with probability $p_{i}$. The density operator of such a system is defined as

$$
\rho=\sum_{i} p_{i}\left|\psi_{i}\right\rangle\left\langle\psi_{i}\right|
$$

The von Neumann entropy [15] $H_{N}$ of a density operator $\rho$ is defined as

$$
H_{N}=-\operatorname{tr}(\rho \log \rho)=-\sum_{i} \xi_{i} \ln \xi_{i}
$$

where $\xi_{1}, \ldots, \xi_{n}$ are the eigenvalues of $\rho$. If $\left\langle\psi_{i}|\rho| \psi_{i}\right\rangle=1$, i.e., the quantum system is a pure state $\left|\psi_{i}\right\rangle$ with probability $p_{i}=1$, then the Von Neumann entropy $H_{N}(\rho)=-\operatorname{tr}(\rho \log \rho)$ is zero. On other hand, for a mixed state described by the density operator $\sigma$ we have a non zero Von Neumann entropy associated with it.

With the Von Neumann entropy to hand, the quantum Jensen-Shannon divergence between two density operators $\rho$ and $\sigma$ is defined as

$$
D_{J S}(\rho, \sigma)=H_{N}\left(\frac{\rho+\sigma}{2}\right)-\frac{1}{2} H_{N}(\rho)-\frac{1}{2} H_{N}(\sigma)
$$

This quantity is always well defined, symmetric and positive definite. Finally, it can also be shown that $D_{J S}(\rho, \sigma)$ is bounded, i.e., $0 \leq D_{J S}(\rho, \sigma) \leq 1$.

\section{QJSD Centrality}

In order to measure the centrality of vertex $v$, we define two quantum walks where $v$ is initially set to be in phase and in antiphase with the respect to the remaining nodes. Let the normalised graph Laplacian be the Hamiltonian of our system, and let $\left|\psi_{0}^{v-}\right\rangle=\sum_{u \in V} \alpha_{u}^{v-}(0)|u\rangle$ and $\left|\psi_{0}^{v+}\right\rangle=\sum_{u \in V} \alpha_{u}^{v+}(0)|u\rangle$ denote the quantum walks on $G$ with initial amplitudes

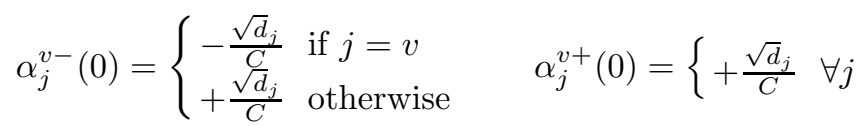


where $C$ is the normalisation constant such that probabilities sum to 1 . In other words, we define the initial amplitude to be proportional to the square root of the node degrees. Finally, let $\rho_{v^{+}}$and $\rho_{v^{-}}$be the density operators which describe the ensembles of quantum states $\left|\psi_{t}^{v-}\right\rangle$ and $\left|\psi_{t}^{v+}\right\rangle$ respectively, i.e.,

$$
\rho_{v^{-}}=\lim _{T \rightarrow \infty} \frac{1}{T} \int_{0}^{T}\left|\psi_{t}^{v-}\right\rangle\left\langle\psi_{t}^{v-}\left|\mathrm{d} t \quad \rho_{v^{+}}=\lim _{T \rightarrow \infty} \frac{1}{T} \int_{0}^{T}\right| \psi_{t}^{v+}\right\rangle\left\langle\psi_{t}^{v+}\right| \mathrm{d} t
$$

Given this setting, we can measure how the initial phase of the vertex $v$ affects the evolution of the quantum walks by computing the distance between the quantum states defined by $\rho_{v^{-}}$and $\rho_{v^{+}}$. That is, we define the quantum Jensen-Shannon divergence (QJSD) centrality of a vertex $v$ as

$$
C_{Q J S D}(v)=D_{J S}\left(\rho_{v^{-}}, \rho_{v^{+}}\right)
$$

Note that the computational complexity of the QJSD centrality is bounded by that of computing the eigendecomposition of the graph laplacian, i.e., $O\left(n^{3}\right)$. Let $\Phi \Lambda \Phi^{\top}$ be the spectral decomposition of the graph normalised Laplacian and let $P_{\lambda}=\sum_{k=1}^{\mu(\lambda)} \phi_{\lambda, k} \phi_{\lambda, k}^{\top}$ be the projection operator on the subspace spanned by the $\mu(\lambda)$ eigenvectors $\phi_{\lambda, k}$ associated with the eigenvalue $\lambda$ of the graph normalised Laplacian. Rossi et al. [16] have shown that $\rho_{\infty}=\sum_{\lambda=1}^{m} P_{\lambda} \rho_{0} P_{\lambda}^{\top}$, where $m$ denotes the number of unique eigenvalues of the graph normalised Laplacian. Note that as a consequence of Eq. 9] we have that $\rho_{v^{-}}$and $\rho_{v^{+}}$are simultaneously diagonalisable. That is, there exist a single invertible matrix $M$ such that $M^{-1} \rho_{v^{-}} M$ and $M^{-1} \rho_{v^{+}} M$ are diagonal. More precisely, here $M=\Phi$, the $n \times n$ matrix $\Phi=\left(\phi_{1}\left|\phi_{2}\right| \ldots\left|\phi_{j}\right| \ldots \mid \phi_{n}\right)$ with the ordered eigenvectors $\phi_{j} \mathrm{~s}$ of the Hamiltonian as columns.

\subsection{Relation with Degree Centrality}

We are now interested in studying the relation between the QJSD centrality and the degree centrality. It has been shown, for example, that the degree and the betweenness centrality are highly correlated [17]. This should not come as a surprise, as we expect high degree vertices to be more often included in the shortest path along a pairs of vertices.

Let the initial states of the walks be defined as in Eq. 8 and let the normalised Laplacian be the Hamiltonian of our system. We start by observing that $\left|\psi_{0}^{v+}\right\rangle=$ $\sum_{u \in V} \alpha_{u}^{v+}(0)|u\rangle$ corresponds to the eigenvector $\phi_{0}$ associated with the zero eigenvalue of the Hamiltonian, and as a consequence $\left|\psi_{0}^{v+}\right\rangle$ will remain constant over time. In other words, we have that $\rho_{v^{+}}=\left|\psi_{0}^{v+}\right\rangle\left\langle\psi_{0}^{v+}\right|$. Note that the spectrum of $\rho_{v^{+}}$is composed of a single eigenvector $\phi_{0}$ with eigenvalue equal to 1. Moreover, recall from Eq9 that $\rho_{v^{-}}$and $\rho_{v^{+}}$are co-diagonalisable matrices. As a result, each eigenvalue of $\rho_{v^{-}}+\rho_{v^{+}}$is a sum of eigenvalues of $\rho_{v^{-}}$and $\rho_{v^{+}}$. More precisely, when the two walks are initialised as in Eq. 8, all the eigenvalues $\mu_{i}$ of $\frac{\rho_{v^{-}}+\rho_{v^{+}}}{2}$ will be equal to the eigenvalues of $\rho_{v^{-}}$, except for the eigenvalue $\mu_{0}+1$ which is associated to the common eigenvector $\phi_{0}$. 


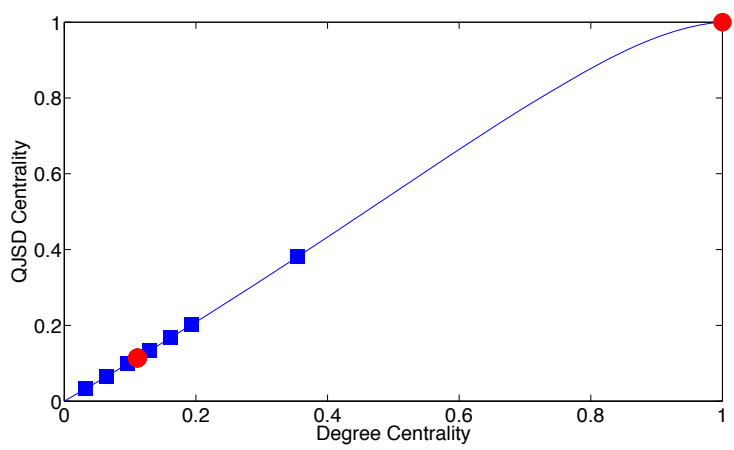

Fig. 1. The correlation between degree and QJSD centrality, for a star graph (red dots) and a scale-free graph (blue squares). The blue line shows the predicted dependency between the two centrality indices.

We now show that, as a consequence of this, the QJSD centrality is proportional to the degree centrality. Note that since $\rho_{v^{+}}$has a single non-zero eigenvalue which is equal to 1 , we have that $H_{N}\left(\rho_{v^{+}}\right)=0$. As a consequence of this and of Eq. 7, we have that

$$
\begin{aligned}
& D_{J S}\left(\rho_{v^{-}}, \rho_{v^{+}}\right)=H_{N}\left(\frac{\rho_{v^{-}}+\rho_{v^{+}}}{2}\right)-\frac{1}{2} H_{N}\left(\rho_{v^{-}}\right) \\
& =-\frac{\mu_{0}+1}{2} \log _{2} \frac{\mu_{0}+1}{2}-\sum_{i \neq 0} \frac{\mu_{i}}{2} \log _{2} \frac{\mu_{i}}{2}+\frac{1}{2} \sum_{i} \mu_{i} \log _{2} \mu_{i} \\
& =\frac{\mu_{0}+1}{2}-\frac{\mu_{0}+1}{2} \log _{2}\left(\mu_{0}+1\right)+\sum_{i \neq 0} \frac{\mu_{i}}{2}-\frac{1}{2} \sum_{i \neq 0} \mu_{i} \log _{2} \mu_{i}+\frac{1}{2} \sum_{i} \mu_{i} \log _{2} \mu_{i} \\
& =1-\frac{1}{2} \log _{2}\left(\mu_{0}+1\right)+\frac{\mu_{0}}{2} \log _{2} \frac{\mu_{0}}{\mu_{0}+1}
\end{aligned}
$$

where $\mu_{i}$ denotes the $i$ th eigenvalue of $\rho_{v^{-}}$and we used the fact that $\sum_{i} \mu_{i}=1$. We now proceed to show that $\mu_{0}$ is proportional to the degree of node $v$, and therefore the QJSD centrality is proportional to the degree centrality. In fact, we have that

$$
\mu_{0}=\left\langle\phi_{0}\left|\rho_{0}\right| \phi_{0}\right\rangle=\left\langle\phi_{0} \mid \psi_{0}^{v-}\right\rangle^{2}=\left(1-\frac{d_{v}}{|E|}\right)^{2}
$$

where $d_{v}$ is the degree of $v$ and $|E|$ denotes the number of edges in the graph.

In other words, when we take the normalised Laplacian as our Hamiltonian and we initialise the walks according to Eq.8, the QJSD centrality turns out to be quasi-linearly correlated with the degree centrality. Fig. 1 shows the correlation between the QJSD centrality and the degree centrality for a scale-free random graph and a star graph. Recall that the degree centrality is normalised between 0 and 1 by dividing it by $|V|(|V|-1)$, i.e., the maximum cardinality of the 


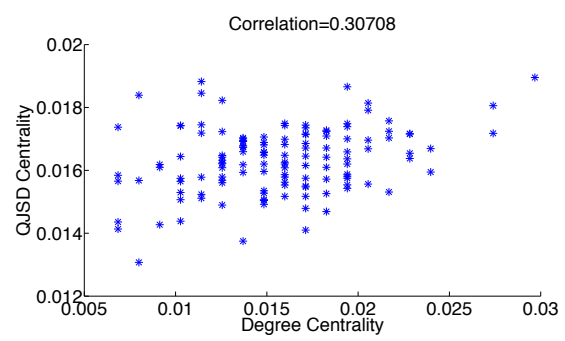

(a) Adjacency + Uniform

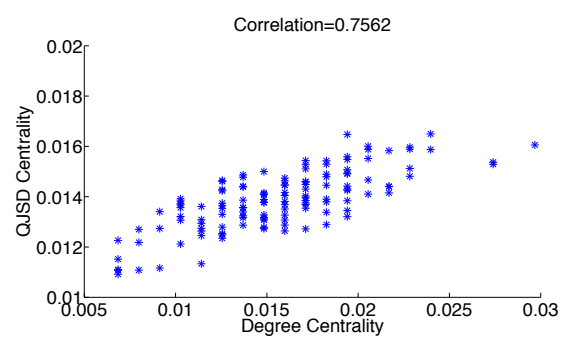

(c) NLap + Uniform

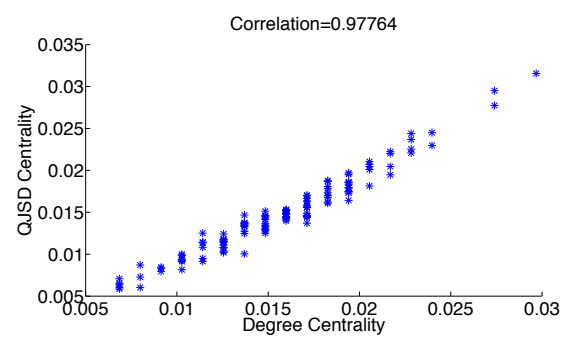

(b) Adjacency + Degree

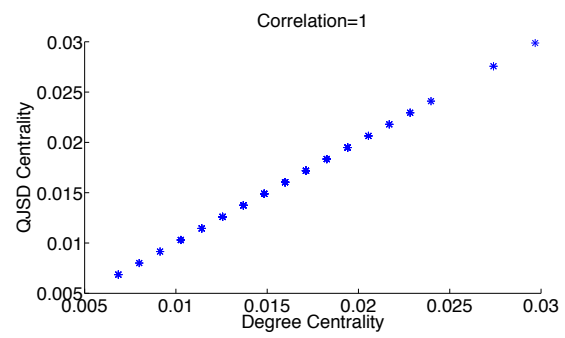

(d) NLap + Degree

Fig. 2. Correlation between the QJSD centrality and the degree centrality for different choices of the Hamiltonian (adjacency matrix or normalised Laplacian) and of the initial state (normalised uniform distribution or normalised degree distribution)

edge set. Note that the non-linearity of the correlation becomes evident only for those nodes with degree close to $|E|$, for which we have that $\frac{d_{v}}{|E|} \approx 0$ and thus $\mu_{0} \approx 1+{\frac{d_{v}}{|E|}}^{2}$.

So far we assumed that the Hamiltonian of the quantum walk is the graph normalised Laplacian. However, any Hermitian operator encoding the structure of the graph can be chosen as an alternative. Similarly, there is no constraint on the initial state of the walk, as long as it is a valid amplitude vector. Fig. 2 shows the correlation between the QJSD centrality and the degree centrality computed on a stochastic Kronecker graph for different choices of the initial state and the Hamiltonian. More specifically, we let the Hamiltonian be either the adjacency matrix or the normalised Laplacian of the graph, while the initial state is either proportional to the node degree as in Eq. 8 or uniformly equal to $1 / \sqrt{n}$, where $n$ denotes the number of nodes in the graph. As expected, our centrality measure is strongly correlated with the degree centrality when the Hamiltonian is the graph normalised Laplacian and the initial state is proportional to the node degree (see Fig. 2(d) . In general, we see that when the starting state is proportional to the node degree, the correlation tends to be very high, while the choice of a uniform initial state leads to a value of the centrality which is less dependent on the node degree.

Hence, in an attempt to capture structural information which are not trivially revealed by examining the node degree, we explore the consequences of letting the 


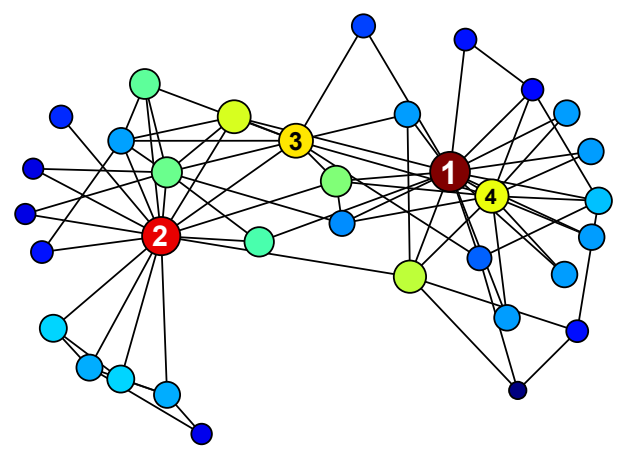

Fig. 3. Zachary's karate club network, where we have drawn each node with a diameter that is proportional to its QJSD centrality

walk start from a uniform amplitude vector and choosing the adjacency matrix as the Hamiltonian. Moreover, in order to balance the strength of the positive and negative signals, i.e., the contribution of the node amplitudes with either positive or negative phases, we let the magnitude of the initial amplitude on the node being analysed be equal to the sum of the amplitudes on the remaining nodes, which gives the initial state

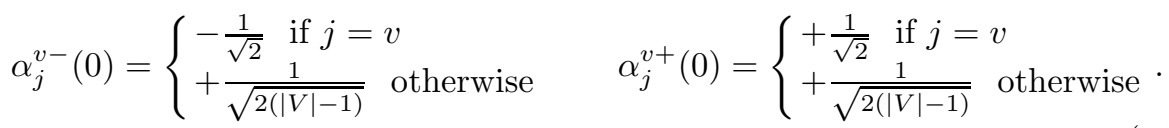

\section{Experimental Evaluation}

We now apply the QJSD centrality to a pair of commonly used network datasets, namely Zachary's karate club [18] and Padgett's network of marriages between the 16 most eminent Florentine families in the 15th century [19]. Fig. 3] shows Zachary's karate club network, where each vertex is drawn with a diameter that is proportional to the QJSD centrality. We see that there are two main actors, node \#1 and node \#2, which correspond to the instructor and the administrator of the club. Note that using our measure the instructor turns out to be the node with the highest centrality, which is also the most central according to the degree centrality, while the betweeness centrality elects the administrator as the most important node. However, the betweenness centrality indicates as the second most important actor node \#3, as this vertex has many contacts with both the members of the administrator cluster and the members of the instructor cluster and thus it is misunderstood as a center by the betweenness centrality. Finally, node \#4 is identified as the third most important by the degree centrality, leaving node \#3 at the fourth place, although the latter is more central in the sense that it shares many links with both groups.

Padgett's network of marriages is depicted in Fig. 4. In Table 1, we show the ranking of the 15 families according to their QJSD centrality. As expected, the 


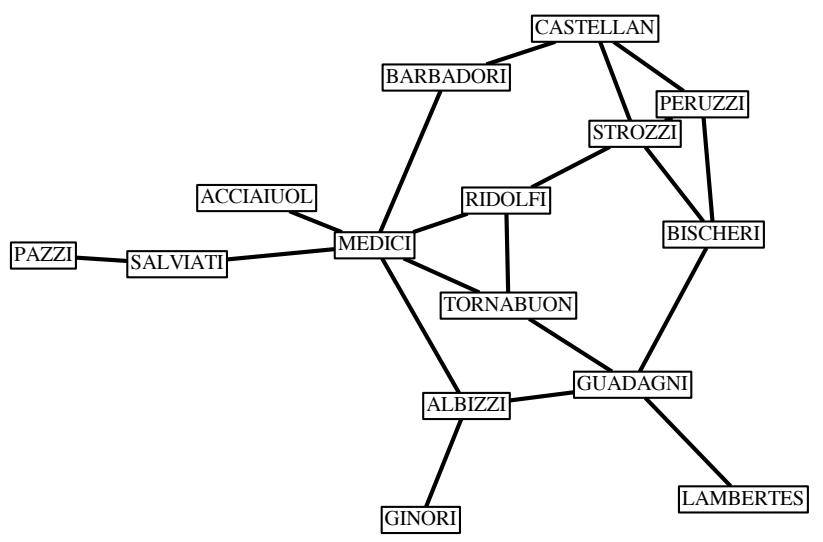

Fig. 4. Padgett's network of marriages between eminent Florentine families in the 15th century [19]. We omit the Pucci, which had no marriage ties with other families.

Table 1. The QJSD centrality of the families of Padgett's network 19]

\begin{tabular}{l|c||l|c||l|c} 
Family & Centrality & Family & Centrality & Family & Centrality \\
\hline Medici & 0.4867 & Castellan & 0.3245 & Salviati & 0.2248 \\
Ridolfi & 0.4619 & Barbadori & 0.3205 & Ginori & 0.1993 \\
Strozzi & 0.4192 & Albizzi & 0.3172 & Acciaiuol & 0.1534 \\
Tornabuon & 0.4041 & Guadagni & 0.3091 & Lambertes & 0.1267 \\
Bischeri & 0.3586 & Peruzzi & 0.2990 & Pazzi & 0.1126
\end{tabular}

Medici easily outperform the Strozzi, who are their main rivals. This agrees with the historical view that Medici's supremacy was largely due to their skills in manipulating the marriage network. Interestingly the Pazzi, which is the most loosely connected family of the graph, achieve the lowest centrality. Note also that the Ridolfi family, which connect two of the most influential families at that time, the Medici and the Strozzi, is assigned a high centrality. Moreover, the Tornabuon, which form a tightly connected clique together with the Medici and the Ridolfi, is the fourth most central node of the network.

\section{Conclusions}

In this paper, we have proposed to measure vertex centrality using a continuoustime quantum walk. We measured the importance of a vertex as the influence that its initial phase has on the interference patterns that emerge during the quantum walk evolution. We have showed that, under particular settings, the resulting centrality measure is almost linearly correlated with degree centrality. Thus, we have proposed an alternative starting state where the contribution of the node amplitudes with positive and negative phases is equal. Finally, we have evaluated the resulting measure to two commonly used network models. 
Acknowledgments. Edwin Hancock was supported by a Royal Society Wolfson Research Merit Award.

\section{References}

1. Estrada, E.: The Structure of Complex Networks. Oxford University Press (2011)

2. Jeong, H., Tombor, B., Albert, R., Oltvai, Z., Barabási, A.: The large-scale organization of metabolic networks. Nature 407, 651-654 (2000)

3. Ito, T., Chiba, T., Ozawa, R., Yoshida, M., Hattori, M., Sakaki, Y.: A comprehensive two-hybrid analysis to explore the yeast protein interactome. Proceedings of the National Academy of Sciences 98, 4569 (2001)

4. Sporns, O.: Network analysis, complexity, and brain function. Complexity 8, 56-60 (2002)

5. Newman, M.: Scientific collaboration networks. i. network construction and fundamental results. Physical Review E 64, 016131 (2001)

6. Freeman, L.C.: A set of measures of centrality based on betweenness. Sociometry, 35-41 (1977)

7. Freeman, L.C.: Centrality in social networks conceptual clarification. Social Networks 1, 215-239 (1979)

8. Newman, M.E.: A measure of betweenness centrality based on random walks. Social Networks 27, 39-54 (2005)

9. Bonacich, P.: Power and centrality: A family of measures. American Journal of Sociology, 1170-1182 (1987)

10. Stanley, W., Faust, K.: Social network analysis: methods and applications. Cambridge University, Cambridge (1994)

11. Kempe, J.: Quantum random walks: an introductory overview. Contemporary Physics 44, 307-327 (2003)

12. Lamberti, P., Majtey, A., Borras, A., Casas, M., Plastino, A.: Metric character of the quantum jensen-shannon divergence. Physical Review A 77, 052311 (2008)

13. Lin, J.: Divergence measures based on the shannon entropy. IEEE Transactions on Information Theory 37, 145-151 (1991)

14. Farhi, E., Gutmann, S.: Quantum computation and decision trees. Physical Review A 58, 915 (1998)

15. Nielsen, M.A., Chuang, I.L.: Quantum computation and quantum information. Cambridge University Press, Cambridge (2010)

16. Rossi, L., Torsello, A., Hancock, E.R., Wilson, R.C.: Characterizing graph symmetries through quantum jensen-shannon divergence. Physical Review E 88, 032806 (2013)

17. Lee, C.Y.: Correlations among centrality measures in complex networks. arXiv preprint physics/0605220 (2006)

18. Zachary, W.: An information flow model for conflict and fission in small groups. Journal of Anthropological Research 33, 452-473 (1977)

19. Padgett, J.F., Ansell, C.K.: Robust action and the rise of the medici, 1400-1434. American Journal of Sociology, 1259-1319 (1993) 